\title{
(2) OPEN ACCESS \\ Effects of angiotensin II receptor blockers on serum potassium level and hyperkalemia risk: retrospective single-centre analysis
}

\author{
Sakae Fukushima, ${ }^{1}$ Manami Oishi, ${ }^{2}$ Hiroya Aso, ${ }^{2}$ Kifumi Arai, ${ }^{3}$ Yuuki Sasaki, ${ }^{3}$ \\ Naohiro Tochikura, ${ }^{1}$ Susumu Ootsuka, ${ }^{1}$ Noriyasu Fukuoka, ${ }^{2}$ Nobuhiro Ooba, ${ }^{2}$ \\ Norikazu Kikuchi ${ }^{3}$
}

\begin{abstract}
PPharmacy, Nihon University Itabashi Hospital, Itabashi-ku, Tokyo, Japan

${ }^{2}$ Nihon University School of Pharmacy, Funabashi, Chiba, Japan

${ }^{3}$ Pharmacy, Nihon University Hospital, Chiyoda-ku, Tokyo, Japan
\end{abstract}

\section{Correspondence to} Sakae Fukushima, Pharmacy, Nihon University Itabashi Hospital, Itabashi-ku 1738610, Tokyo, Japan; fukushima. sakae@nihon-u.ac.jp

Received 15 February 2021 Accepted 15 June 2021

\section{EAHP Statement 4: Clinical} Pharmacy Services.
D) Check for updates

(c) European Association of Hospital Pharmacists 2021. Re-use permitted under CC BY-NC. No commercial re-use. Published by BMJ.

\section{To cite: Fukushima $\mathrm{S}$, Oishi M, Aso H, et al. Eur I Hosp Pharm Epub ahead of print: [please include Day Month Year]. doi:10.1136/ \\ ejhpharm-2021-002739}

\begin{abstract}
Objective To examine the effect of angiotensin II receptor blocker (ARB) treatment on serum potassium level and hyperkalaemia risk in a clinical setting with inpatients and outpatients using calcium channel blockers (CCBs) as a reference standard.

Methods The increased risk of hyperkalaemia associated with ARB treatment is known, however only a few studies have used an active comparator to examine this risk. In this retrospective study at a 320-bed general hospital in Japan, the hospital information system was used to identify patients with at least one prescription for an ARB (819 patients) or a CCB (1015 patients) who were naive to these drugs before study initiation. Serum potassium levels before and after ARB treatment were compared. Additionally, the unadjusted and adjusted hazard ratios for the risk of hyperkalaemia in the ARB and CCB users were estimated.
\end{abstract}

Results The serum potassium level was higher in patients receiving $A R B$ treatment $(0.05 \mathrm{mEq} / \mathrm{L}, \mathrm{p}=0.02)$ compared with those on CCB treatment. However, there was no significant association between ARB use and hyperkalaemia (adjusted HR 0.91, 95\% Cl 0.42 to 1.99 , $\mathrm{p}=0.82$ ).

Conclusion The increase in serum potassium level after ARB initiation makes it necessary to monitor serum potassium levels continuously during ARB treatment; however, the risk of hyperkalaemia appeared to be similar for ARB and CCB treatments.

\section{INTRODUCTION}

Hypertension is a well known risk factor for coronary heart disease, stroke, transient ischaemic attack, and diabetes-induced chronic kidney disease. ${ }^{1-4}$ The incidence and prevalence of hypertension are associated with age, ${ }^{5}$ therefore the number of patients with hypertension will probably increase in the future. Blood pressure control and hypertension management are essential to prevent hypertension-related complications.

Few guidelines for hypertension recommend angiotensin II receptor blockers (ARBs) as the first drug of choice for treating hypertension. ${ }^{2-4}$ In Japan, ARBs and calcium channel blockers (CCBs) are commonly used as antihypertensive agents in patients of all ages ${ }^{7}$; however, the choice of frequently used drugs varies among countries. ${ }^{8-12}$ The advantages of ARBs are as follows: reduction in proteinuria ${ }^{13}$ and protection against the progression of type 2 diabetes induced nephropathy that is independent of their effect on blood pressure. ${ }^{14-16}$ The influence on heart failure might not be a class effect of $\mathrm{ARBs}^{17}$; however, the ARB losartan reduced the risk by $32 \% .^{15}$ Losartan reduces uric acid (UA) levels, ${ }^{18-21}$ but ARBs, including losartan, ${ }^{22}$ increase the risk of hyperkalaemia. ${ }^{23}$ Therefore, monitoring the levels of serum potassium and creatinine is recommended for patients on ARBs. ${ }^{23}$ Fang and colleagues $^{25}$ reported a low incidence of hyperkalaemia $(0.5 \%)$ in patients aged 66 years and older using data from the Medicare service claims database and a drug database of the United States. However, a study in Sweden reported a relatively high incidence proportion of hyperkalaemia (5.6\%) in new users of ARBs. ${ }^{26}$ Although surveillance of treatment-related hyperkalaemia is important, ${ }^{27}$ only a few studies ${ }^{15} 28$ have used an active comparator, such as CCBs, to examine the role ARBs have in hyperkalaemia. The cumulative incidence rate of hyperkalaemia increases from $0.3 \%$ to $0.5 \%$ in older patients after 6 months of treatment with angiotensin-converting enzyme inhibitors or ARBs. ${ }^{25}$ Hyperkalaemia occurs frequently in the first week after ARB initiation. ${ }^{23}$ A few randomised controlled trials on ARB with a follow-up period of approximately 3 years indicated that the risk of hyperkalaemia increases, ${ }^{15} 29$ while others have reported no association. ${ }^{30-32}$ Therefore, it is necessary to elucidate the risk of hyperkalaemia in patients with long-term follow-up.

The objective of our retrospective study was to assess the risk of hyperkalaemia in response to ARB treatment. The serum levels of potassium and uric acid in response to ARB or CCB treatment were examined in a clinical setting. The findings of this study can provide evidence of the risk of hyperkalaemia in patients prescribed ARBs.

\section{METHODS}

\section{Setting and population}

We used the claims data and laboratory test results obtained from Nihon University Hospital (Tokyo, Japan), a 320-bed general hospital. Inpatients and outpatients with at least one prescription for ARBs or CCBs between 1 October 2014 and 30 June 2018 were included in this study.

\section{Study design}

This is a retrospective cohort study. The data for the study were collected from the hospital information 
system for individual patients who were prescribed ARBs or CCBs (used as the reference standard). The details collected were patient study ID number (created by a researcher in the hospital); patient demographics (age and sex); dates of hospital visits; diagnoses, as coded using the International Classification of Diseases, 10th edition (ICD-10); laboratory test orders for serum UA, potassium, and creatinine levels; laboratory test values, including the serum UA $(\mathrm{mg} / \mathrm{dL})$ and serum potassium $(\mathrm{mEq} / \mathrm{L})$ levels; generic names of the drugs; the date of initiation and number of days of administration of the prescribed drugs.

The index date was defined as the day on which the study drug was initiated. The baseline period was defined as the period after the commencement of hospital visits, but before the index date. The observation period was defined as the period from the index date to the last prescription date of each study drug plus the number of prescription days.

All patients who had been prescribed at least one of the study drugs (ie, oral dosage forms of ARBs or CCBs) during the study period were initially included in the study. Patients were excluded if they had received two or more study drugs or if they were concurrently prescribed other antihypertensives on the index date. To mitigate bias created by long-term use, we restricted the study to patients who were naive to the study drugs, based on the record of hospital visits. ${ }^{33}$ Hospital researchers identified patients who were initially prescribed ARBs or CCBs between October 2014 and April 2015 based on the data of their hospital visits. Patients with a baseline period shorter than 6 months and/ or those who received any antihypertensives during the 6 month period before the index date were excluded from the study.

\section{Outcomes}

The primary outcome was the association between hyperkalaemia and ARB treatment compared with the association with CCB treatment. The time to the first event during follow-up was estimated using the Kaplan-Meier plot. Secondary outcomes were the differences in the serum potassium and UA levels before and after ARB or CCB initiation.

\section{Statistical analyses}

We assessed the summary statistics of demographic characteristics, comorbidities, co-medications, and laboratory tests of study patients at the baseline period. The number and proportion of patients with these covariates are shown. To compare the baseline characteristics, we calculated the standardised differences among variables. Standardised differences greater than 0.1 were considered meaningful. ${ }^{34}$

We defined the occurrence of hyperkalaemia based on the diagnostic code of hyperkalaemia (E875, ICD-10 code) and the laboratory test order for serum potassium in the claims data. We calculated the period from ARB or CCB initiation to the occurrence of hyperkalaemia. The diagnostic code for hyperkalaemia was used to compare the incidence proportion of hyperkalaemia among patients receiving ARBs with that in those receiving CCBs. The time to the occurrence of hyperkalaemia was depicted using a Kaplan-Meier curve and compared using a log-rank test. We estimated the HR and its 95\% CI for the incidence proportion of hyperkalaemia using the Cox proportional hazards model. The incidence proportion of hyperkalaemia in patients receiving CCB treatment was used as the reference standard. Unadjusted and adjusted HRs were calculated in patients without a history of hyperkalaemia. Using the Cox proportional hazards model, we adjusted for age, sex, and known risk factors for hyperkalaemia (renal diseases, heart failure, and diabetes). ${ }^{23} 35$

The differences in serum levels of potassium and UA during the 3 month period before and after the index date were compared using a paired $t$-test by drug groups. The mean difference between serum potassium levels before and after the start of ARBs and CCBs was compared using a two-sample $t$-test after applying the $F$-test for equality of variance.

To confirm the validity of using the diagnostic code ICD-10 E875 as an outcome, we collected data for all patients with a code of ICD-10 E875 between January 2017 and December 2017, regardless of whether they had used ARBs or CCBs. We used serum potassium level to determine the positive predictive value (PPV) of this code. Hyperkalaemia is defined as a serum

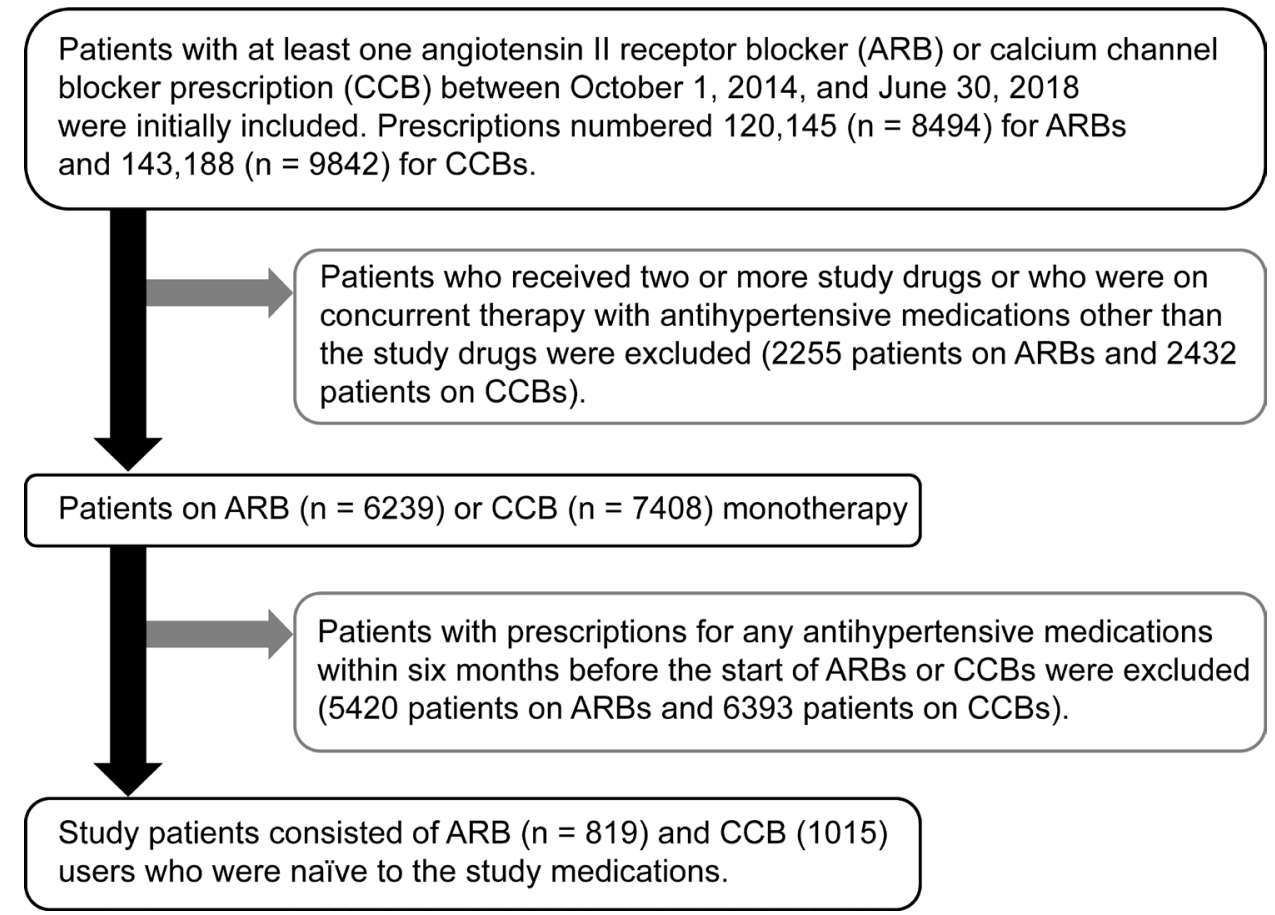

Figure 1 Study flow diagram for patient selection. $A R B=$ angiotensin II receptor blocker; $C C B=$ calcium channel blocker. 
Table 1 Baseline characteristics of patients. Values are numbers (\%) unless indicated otherwise.

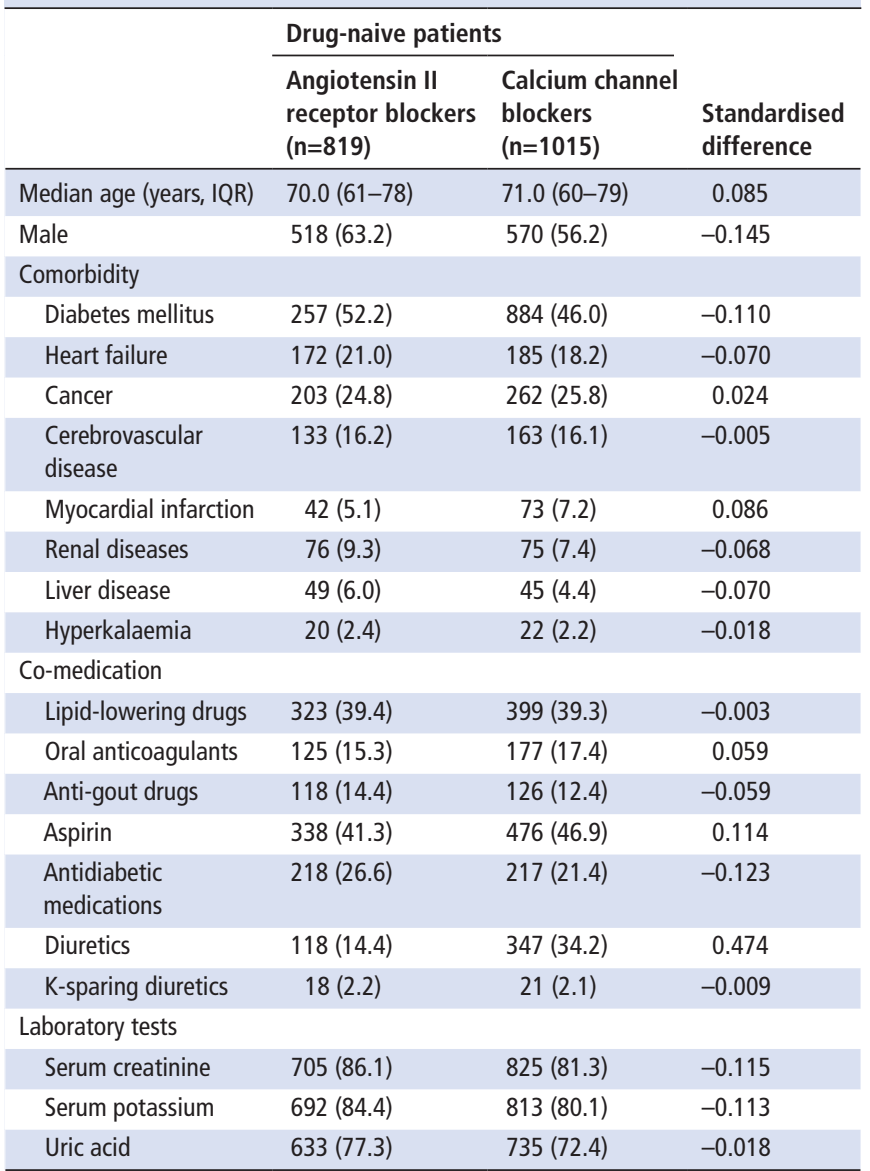

Lipid-lowering drugs include statins and fibrates. Anti-gout drugs include antihyperuricaemic drugs.

$I Q R$, interquartile range.

potassium level of either $\geq 5$ or $\geq 5.5 \mathrm{mEq} / \mathrm{L} .{ }^{35}$ We used these values and the relatively recent data (from 2017) to estimate the PPV and its 95\% CI for the condition code of hyperkalaemia.

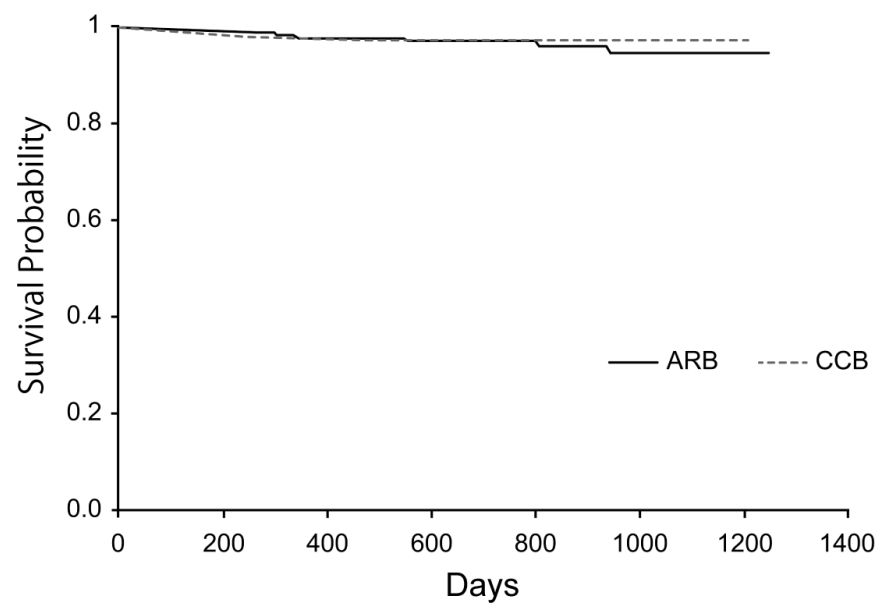

Figure 2 Probability of hyperkalaemia among patients receving angiotensin II receptor blocker (ARBS) and calcium channel blocker (CCBs). Survival probability at a time point is the unadjusted probability of not developing hyperkalaemia by that time point. $A R B=$ angiotensin II receptor blocker; $\mathrm{CCB}=$ calcium channel blocker.

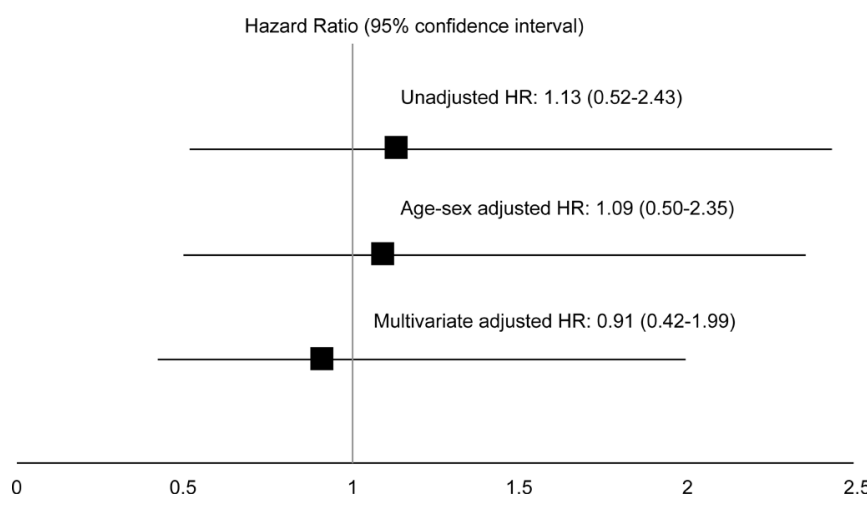

Figure 3 Unadjusted and adjusted hazard ratios for hyperkalaemia in the angiotensin II receptor blocker (ARB) group compared with those in the calcium channel blocker (CRB) group. Multivariate adjusted HR included covariates of age, sex, diabetes, chronic heart failure, renal disease, antidiabetic use, and laboratory test for potassium level.

Results with a p value $<0.05$ were considered statistically significant. All analyses were conducted using SAS version 9.4 (SAS Institute, Cary, NC, USA).

\section{RESULTS}

\section{Baseline characteristics}

The number of prescriptions for ARBs and CCBs between 1 October 2014 and 30 June 2018 was $120145(\mathrm{n}=8494)$ and 143188 ( $n=9842)$, respectively. Of those, the number of ARBnaive users was 819 (9.6\% of ARB users) and the number of CCB-naive users was 1015 (10.4\% of CCB users). The flow diagram for the selection of ARB-naive and CCB-naive patients is shown in figure 1 .

The mean age $\pm S D$ of patients treated with $\mathrm{ARBs}$ was $67.4 \pm 15.5$ years, and that of patients treated with CCBs was $68.7 \pm 13.1$ years. The proportion of men was $63.2 \%$ in the ARB group and $56.2 \%$ in the CCB group. Some variables were dissimilar between the groups; standardised differences exceeded 0.1 for characteristics, such as sex, diabetes mellitus, and antidiabetic drug use. The characteristics of patients who were naive to the study drugs are listed in table 1 .

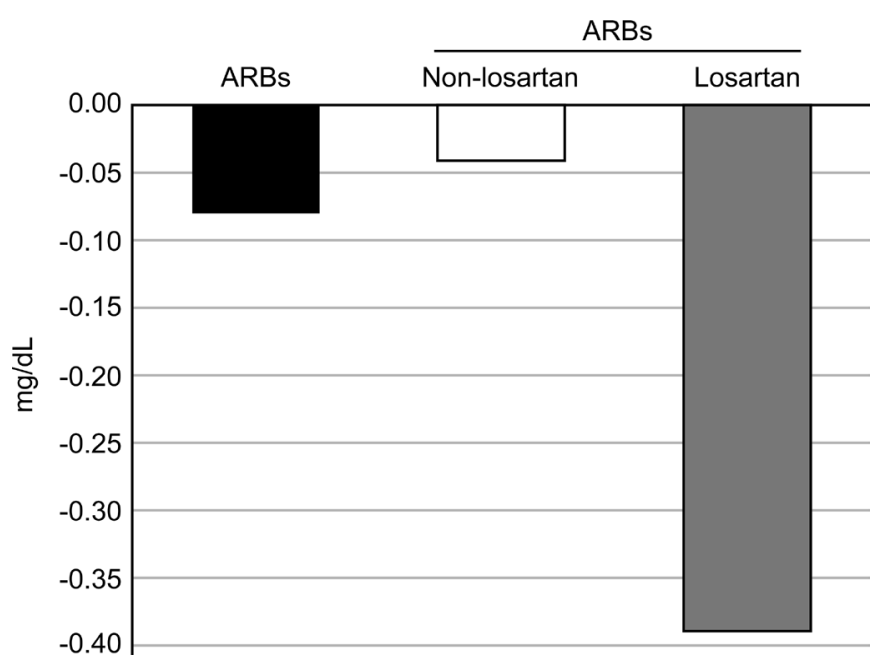

Figure 4 Differences in uric acid levels before and after angiotensin II receptor blocker (ARB) initiation. 
Table 2 Positive predictive value of the diagnostic code for hyperkalaemia in 2017

No of patients with hyperkalaemia as defined by laboratory test value $(\%$, $95 \% \mathrm{Cl})$

\begin{tabular}{|c|c|c|c|}
\hline \multirow[b]{2}{*}{ Non-laboratory parameters for defining hyperkalaemia } & \multirow[b]{2}{*}{ Total } & \\
\hline & & Serum potassium $\geq 5 \mathrm{mEq} / \mathrm{L}$ & Serum potassium $\geq 5.5 \mathrm{mEq} / \mathrm{L}$ \\
\hline ICD-10 E875 & 149 & $\begin{array}{l}109 \\
(73.2,66.0 \text { to } 80.3)\end{array}$ & $\begin{array}{l}64 \\
(43.0,35.0 \text { to } 50.9)\end{array}$ \\
\hline ICD-10 E875+laboratory test order for serum potassium & 129 & $\begin{array}{l}109 \\
(85.0,78.3 \text { to } 90.7)\end{array}$ & $\begin{array}{l}64 \\
(49.6,41.0 \text { to } 58.2)\end{array}$ \\
\hline
\end{tabular}

\section{Hyperkalaemia risk of ARBs}

Figure 2 shows the Kaplan-Meier curves for hyperkalaemia during drug use. A log-rank test revealed that the time to hyperkalaemia did not significantly differ between patients treated with ARBs and those treated with CCBs $(p=0.76)$. The number of patients with hyperkalaemia was 13 each in the ARB (incidence proportion $0.02 ; 95 \% \mathrm{CI} 0.01$ to 0.03 ) and $\mathrm{CCB}$ (incidence proportion $0.01 ; 95 \% \mathrm{CI} 0.01$ to 0.02 ) groups. The median time (IQR) from ARB or CCB initiation to the occurrence of hyperkalaemia was 230 (59-343) days for ARBs and 150 (81-208) days for CCBs. Based on the incidence proportion of hyperkalaemia for CCBs as a reference, the difference in the unadjusted HR $(1.13,95 \%$ CI 0.52 to $2.43, p=0.76)$ and adjusted HR (0.91, $95 \%$ CI 0.42 to $1.99, \mathrm{p}=0.82$ ) for hyperkalaemia between ARB and CCB users was not significant (figure 3 ).

\section{Changes in serum potassium and serum UA levels after ARB use}

The difference in serum potassium levels before and after the index date was $0.03 \mathrm{mEq} / \mathrm{L}(95 \% \mathrm{CI}-0.004$ to $0.07 \mathrm{mEq} / \mathrm{L})$ for $\mathrm{ARBs}$ and $-0.02 \mathrm{mEq} / \mathrm{L}(95 \% \mathrm{CI}-0.05$ to $0.003 \mathrm{mEq} / \mathrm{L})$ for CCBs. The mean difference between patients receving ARBs and CCBs was significant with respect to the changes in serum potassium levels before and after the index date $(0.05 \mathrm{mEq} / \mathrm{L}$, 95\% CI 0.01 to $0.10 \mathrm{mEq} / \mathrm{L}, \mathrm{p}=0.01$ ).

The difference in serum UA levels before and after ARB initiation was not significant for ARBs as a class $(-0.08 \mathrm{mg} / \mathrm{dL}$, $95 \% \mathrm{CI}-0.19$ to $0.02 \mathrm{mg} / \mathrm{dL}, \mathrm{p}=0.13$ ) or for non-losartan ARBs $(-0.04 \mathrm{mg} / \mathrm{dL}, 95 \% \mathrm{CI}-0.15$ to $0.07 \mathrm{mg} / \mathrm{dL}, \mathrm{p}=0.45$; figure 4$)$. In contrast, the serum UA level was significantly lower after losartan use compared with that before losartan use (mean difference $-0.39 \mathrm{mg} / \mathrm{dL}, 95 \% \mathrm{CI}-0.76$ to $-0.03 \mathrm{mg} / \mathrm{dL}, \mathrm{p}=0.04$ ).

\section{PPV of the condition code for hyperkalaemia}

The PPV of inpatient and outpatient claims code ICD-10 E875 for the diagnosis of hyperkalaemia during 2017 is shown in table 2. When hyperkalaemia was defined by a combination of claims code ICD-10 E875 and laboratory orders for serum potassium level, the PPV was $85.0 \%$ (95\% CI 78.3\% to $90.7 \%$ ) and $49.6 \%$ (95\% CI $41.0 \%$ to $58.2 \%)$ for serum potassium levels of $\geq 5$ and $\geq 5.5 \mathrm{mEq} / \mathrm{L}$, respectively.

\section{DISCUSSION}

In this retrospective study, we used claims data and hospital laboratory test results to examine whether ARBs influence the serum potassium and UA levels. We found that the risk of hyperkalaemia in patients receiving ARBs was similar to that in those receiving $\mathrm{CCBs}$, although an increase in the serum potassium level was observed in those on ARBs.

The association between hyperkalaemia and ARB use is well known. ${ }^{36}$ In our study, the use of ARBs led to an insignificant increase in the serum potassium level. This increase was significant compared with that measured after the initiation of CCBs; however, the incidence proportion of hyperkalaemia was not significantly higher in ARB-naive patients compared with that in CCB-naive patients. The incidence proportion of hyperkalaemia was around $2 \%$ in ARB users; however, the proportion may differ between populations. The incidence proportion in previous studies 25 showed a wide range (0.5-5.6\%); the underlying reason needs to be explored in future studies. A network meta-analysis reported that ARB treatment in patients with type 2 diabetes and chronic kidney disease did not significantly increase the risk of hyperkalaemia (OR 1.88, 95\% CI 0.86 to 4.12$).{ }^{27}$ A nationwide cohort study from 122 centres in Brazil reported that ARBs are not associated with a greater risk of hyperkalaemia in patients with stable disease on peritoneal dialysis. ${ }^{37}$ The differences in some of the covariates (eg, the presence of diabetes, renal disease, and antidiabetic medications; table 1) between patients on ARBs and those on CCBs might have contributed to our findings.

The reduction in the serum levels of UA was not a class effect of ARBs. Non-losartan ARBs had no significant effect on the UA level, but losartan reduced the UA level; this was consistent with the results of previous studies. ${ }^{21} 38-40$ The inhibitory effect of losartan on urate transporter 1 is the potential biological mechanism underlying its anti-uricosuric effects in patients with hypertension. ${ }^{41}$

\section{Strengths}

The strength of this study is the high PPV (85.0\%) for hyperkalaemia of $\geq 5 \mathrm{mEq} / \mathrm{L}$ when the combination of ICD-10 E875 and laboratory orders for serum potassium level was used. The PPV for the potassium level of $\geq 5.5 \mathrm{mEq} / \mathrm{L}$ was low $(49.6 \%$ ); however, we consider the PPV for the serum potassium level of $\geq 5.0 \mathrm{mEq} / \mathrm{L}$ as critical because, in clinical practice, this is the level at which the risk of renal events begins to increase. ${ }^{42}$ Additionally, there are only a few studies with ARBs as an active comparator. ${ }^{1528}$ The design of randomised controlled trials is different from that of our observational study; however, randomised controlled trials have a high internal, but low external validity, whereas observational studies in clinical settings have low internal and high external validity. ${ }^{43}$

\section{Limitations}

Our study had some potential limitations. First, it was conducted in a single centre in Japan; this might restrict the generalisability of our findings, including those for PPVs. Second, the HR for hyperkalaemia might have been unstable because of the wide $95 \% \mathrm{CI}$, which was a result of the small number of patients. Third, we were unable to examine the dose-response relationship because we had no information on the dosage of study drugs.

The serum potassium level was higher after the initiation of ARBs compared with that after the initiation of CCBs; however, a comparison of the incidence proportion of hyperkalaemia between patients receiving $\mathrm{ARBs}$ and those receiving $\mathrm{CCBs}$ 
revealed no association between ARB use and hyperkalaemia. Therefore, it may be important to assess the potassium level and renal function at the start of ARB use. To evaluate the risk of hyperkalaemia, further studies using a higher number of patients in multiple clinical centres are needed.

What this paper adds

What is already known on this subject

- Angiotensin II receptor blockers (ARBs) are drugs of choice for the treatment of hypertension.

- ARBs are known to increase the risk of hyperkalaemia.

- ARB losartan has a beneficial effect of reducing uric acid (UA) level.

\section{What this study adds}

- The risk of hyperkalaemia in patients receiving ARBs was similar to that in patients receiving calcium channel blockers.

- The use of ARBs led to a non-significant increase in serum potassium levels, although this increase was significant compared with that measured after the initiation of CCBs.

- ARBs other than losartan did not reduce the UA level, indicating that it was not a class effect of ARBs.

Contributors All authors contributed to the design and implementation of the research, to the analysis of the results and to the writing of the manuscript.

Funding This work was partially supported by a Nihon University Chairman of the Board of Trustees Grant.

Competing interests None declared.

Patient consent for publication Not required.

Ethics approval This study was approved by the ethics committees of both Nihon University Hospital (no. 20180602) and Nihon University School of Pharmacy (no. 18-009). Both ethics committees waived the need for informed consent from patients, as the study data were fully anonymised.

Provenance and peer review Not commissioned; externally peer reviewed. Data availability statement No data are available. fukushima.sakae@nihon-u. ac.jp.

Open access This is an open access article distributed in accordance with the Creative Commons Attribution Non Commercial (CC BY-NC 4.0) license, which permits others to distribute, remix, adapt, build upon this work non-commercially, and license their derivative works on different terms, provided the original work is properly cited, an indication of whether changes were made, and the use is noncommercial. See: http://creativecommons.org/licenses/by-nc/4.0/.

\section{REFERENCES}

1 Kokubo Y, Matsumoto C. Hypertension is a risk factor for several types of heart disease: review of prospective studies. Adv Exp Med Biol 2017;956:419-26.

2 Umemura S, Arima H, Arima S, et al. The Japanese Society of hypertension guidelines for the management of hypertension (JSH 2019). Hypertens Res 2019:42:1235-481.

3 Whelton PK, Carey RM, Aronow WS, et al. 2017 ACC/AHA/AAPA/ABC/ACPM/AGS/ APhA/ASH/ASPC/NMA/PCNA guideline for the prevention, detection, evaluation, and management of high blood pressure in adults: Executive summary: a report of the American College of Cardiology/American heart association Task force on clinical practice guidelines. Hypertension 2018;71:1269-324.

4 Williams B, Mancia G, Spiering W, et al. 2018 ESC/ESH guidelines for the management of arterial hypertension. Eur Heart J 2018;39:3021-104.

5 Lloyd-Jones DM, Evans JC, Levy D. Hypertension in adults across the age spectrum: current outcomes and control in the community. JAMA 2005:294:466-72.

6 Mosley WJ, Lloyd-Jones DM. Epidemiology of hypertension in the elderly. Clin Geriatr Med 2009;25:179-89.

7 Ishida T, Oh A, Hiroi S, et al. Current use of antihypertensive drugs in Japanese patients with hypertension: analysis by age group. Geriatr Gerontol Int 2018;18:899-906.

8 Hwang AY, Dave C, Smith SM. Trends in antihypertensive medication use among US patients with resistant hypertension, 2008 to 2014. Hypertension 2016:68:1349-54

9 Sundbøll J, Adelborg K, Mansfield KE, et al. Seventeen-Year nationwide trends in antihypertensive drug use in Denmark. Am J Cardiol 2017;120:2193-200.
10 Maio V, Gagne JJ. Impact of ALLHAT publication on antihypertensive prescribing patterns in Regione Emilia-Romagna, Italy. J Clin Pharm Ther 2010;35:55-61.

11 Rudolph UM, Enners S, Kieble M, et al. Impact of angiotensin receptor blocker product recalls on antihypertensive prescribing in Germany. J Hum Hypertens 2020. doi:10.1038/s41371-020-00425-z. [Epub ahead of print: 14 Oct 2020].

12 Huang L-Y, Shau W-Y, Chen H-C, et al. Pattern analysis and variations in the utilization of antihypertensive drugs in Taiwan: a six-year study. Eur Rev Med Pharmacol Sci 2013:17:410-9.

13 Kunz R, Friedrich C, Wolbers M, et al. Meta-Analysis: effect of monotherapy and combination therapy with inhibitors of the renin angiotensin system on proteinuria in renal disease. Ann Intern Med 2008;148:30-48.

14 Brenner BM, Cooper ME, de Zeeuw D, et al. Effects of losartan on renal and cardiovascular outcomes in patients with type 2 diabetes and nephropathy. $N$ Eng/ J Med 2001;345:861-9.

15 Lewis EJ, Hunsicker LG, Clarke WR, et al. Renoprotective effect of the angiotensinreceptor antagonist irbesartan in patients with nephropathy due to type 2 diabetes. $N$ Engl J Med 2001;345:851-60.

16 Parving $\mathrm{HH}$, Lehnert $\mathrm{H}$, Bröchner-Mortensen J, et al. The effect of irbesartan on the development of diabetic nephropathy in patients with type 2 diabetes. $N$ Engl J Med 2001;345:870-8

17 Akioyamen L, Levine M, Sherifali D, et al. Cardiovascular and cerebrovascular outcomes of long-term angiotensin receptor blockade: meta-analyses of trials in essential hypertension. J Am Soc Hypertens 2016;10:55-69.

18 Chida R, Hisauchi I, Toyoda S, et al. Impact of irbesartan, an angiotensin receptor blocker, on uric acid level and oxidative stress in high-risk hypertension patients. Hypertens Res 2015;38:765-9.

19 Dang A, Zhang Y, Liu G, et al. Effects of losartan and irbesartan on serum uric acid in hypertensive patients with hyperuricaemia in Chinese population. J Hum Hypertens 2006;20:45-50.

20 Elliott WJ, Calhoun DA, DeLucca PT, et al. Losartan versus valsartan in the treatment of patients with mild to moderate essential hypertension: data from a multicenter, randomized, double-blind, 12-week trial. Clin Ther 2001;23:1166-79.

21 Rayner BL, Trinder YA, Baines D, et al. Effect of losartan versus candesartan on uric acid, renal function, and fibrinogen in patients with hypertension and hyperuricemia associated with diuretics. Am J Hypertens 2006:19:208-13.

22 Rossignol P, Dobre D, Gregory D, et al. Incident hyperkalemia may be an independent therapeutic target in low ejection fraction heart failure patients: insights from the HEAAL study. Int I Cardiol 2014;173:380-7.

23 Park I-W, Sheen SS, Yoon D, et al. Onset time of hyperkalaemia after angiotensin receptor blocker initiation: when should we start serum potassium monitoring? J Clin Pharm Ther 2014:39:61-8.

24 Raebel MA, McClure DL, Simon SR, et al. Laboratory monitoring of potassium and creatinine in ambulatory patients receiving angiotensin converting enzyme inhibitors and angiotensin receptor blockers. Pharmacoepidemiol Drug Saf 2007;16:55-64.

25 Fang G, Annis IE, Farley JF, et al. Incidence of and risk factors for severe adverse events in elderly patients taking angiotensin-converting enzyme inhibitors or angiotensin II receptor blockers after an acute myocardial infarction. Pharmacotherapy 2018:38:29-41.

26 Bandak G, Sang Y, Gasparini A, et al. Hyperkalemia after initiating renin-angiotensin system blockade: the Stockholm creatinine measurements (scream) project. J Am Heart Assoc 2017;6:e005428.

27 Palmer SC, Mavridis D, Navarese E, et al. Comparative efficacy and safety of blood pressure-lowering agents in adults with diabetes and kidney disease: a network meta-analysis. Lancet 2015;385:2047-56.

28 Formica RN, Friedman AL, Lorber Ml, et al. A randomized trial comparing losartan with amlodipine as initial therapy for hypertension in the early post-transplant period. Nephrol Dial Transplant 2006:21:1389-94.

29 Yusuf S, Pfeffer MA, Swedberg K, et al. Effects of candesartan in patients with chronic heart failure and preserved left-ventricular ejection fraction: the CHARM-Preserved trial. Lancet 2003;362:777-81.

30 NAVIGATOR Study Group, McMurray JJ, Holman RR, et al. Effect of valsartan on the incidence of diabetes and cardiovascular events. N Eng/ J Med 2010;362:1477-90.

31 Menne J, Izzo JL, Ito S, et al. Prevention of microalbuminuria in patients with type 2 diabetes and hypertension. J Hypertens 2012:30:811-8.

32 Massie BM, Carson PE, McMurray JJ, et al. Irbesartan in patients with heart failure and preserved ejection fraction. N Eng/ J Med 2008;359:2456-67.

33 Ray WA. Evaluating medication effects outside of clinical trials: new-user designs. Am J Epidemiol 2003;158:915-20

34 Mamdani M, Sykora K, Li P, et al. Reader's guide to critical appraisal of cohort studies: 2. assessing potential for confounding. BMJ 2005:330:960-2.

35 Lindner G, Burdmann EA, Clase CM, et al. Acute hyperkalemia in the emergency department: a summary from a kidney disease: improving global outcomes conference. Eur J Emerg Med 2020;27:329-37.

36 Raebel MA. Hyperkalemia associated with use of angiotensin-converting enzyme inhibitors and angiotensin receptor blockers. Cardiovasc Ther 2012;30:e156-66.

37 Ribeiro SC, Figueiredo AE, Barretti P, et al. Impact of renin-angiotensin aldosterone system inhibition on serum potassium levels among peritoneal dialysis patients. Am J Nephrol 2017:46:150-5. 


\section{Original research}

38 Fan Y, Wei F, Lang Y, et al. Losartan treatment for hypertensive patients with hyperuricaemia in Chinese population: a meta-analysis. J Hypertens 2015;33:681-9.

39 Naritomi H, Fujita T, Ito S, et al. Efficacy and safety of long-term losartan therapy demonstrated by a prospective observational study in Japanese patients with hypertension: the Japan hypertension evaluation with angiotensin II antagonist losartan therapy (J-HEALTH) study. Hypertens Res 2008;31:295-304.

40 Wolff ML, Cruz JL, Vanderman AJ, et al. The effect of angiotensin II receptor blockers on hyperuricemia. Ther Adv Chronic Dis 2015;6:339-46.
41 Hamada T, Ichida K, Hosoyamada M, et al. Uricosuric action of losartan via the inhibition of urate transporter 1 (URAT 1) in hypertensive patients. Am J Hypertens 2008;21:1157-62.

42 Miao Y, Dobre D, Heerspink HJL, et al. Increased serum potassium affects renal outcomes: a post hoc analysis of the reduction of endpoints in NIDDM with the angiotensin II antagonist losartan (RENAAL) trial. Diabetologia 2011;54:44-50.

43 Michel MC, Goepel M. Treatment satisfaction of patients with lower urinary tract symptoms: randomised controlled trials vs. real life practice. Eur Urol 2000;38 Suppl $1: 40-7$. 